\title{
Swelling/deswelling Mechanism of Calcium Alginate Gel in Aqueous Solutions
}

\author{
Setsuo SAITOH, Yoshima ARAKI, Ryuichi KON, Hirohumi KATSURA \\ and Masayuki TAIRA \\ Department of Dental Materials Science and Technology, \\ Iwate Medical University School of Dentistry \\ 1-3-27, Chuo-dori, Morioka, Iwate 020-8505, Japan
}

Received July 15, 2000/Accepted September 22, 2000

\begin{abstract}
To elucidate the mechanism of dimensional changes in alginate impression in solutions, the relationship between the ion concentrations in three types of solutions (nonelectrolyte and monovalent and divalent metallic salts) and change in gel volume was examined. The gel in the monovalent metallic salt solution expanded and a decrease in monovalent cation and an increase in $\mathrm{Ca}^{2+}$ were observed. This suggests that the crosslinking density of the gel reduced due to dissociation of $\mathrm{Ca}^{2+}$ from the calcium alginate gel. In divalent metallic salt solutions, the shrinkage occurred according to crosslinking of unreacted residue. In the nonelectrolytic solution, gel, neither ethylene glycol nor acetonitrile showed any volume changes, while that in glutaraldehyde contracted. It is speculated that the former two solutions were affected by the fact that the gel has no semipermeability, and that the latter result was due to chemical interaction between the gel and solution.
\end{abstract}

Key words: Calcium alginate, Aqueous solutions, Swelling/deswelling

\section{INTRODUCTION}

There are dimensional changes in alginate impressions in various solutions ${ }^{1-3)}$, and the effects of solutions on surface roughness of stone casts ${ }^{4-6)}$ have been investigated to develop a disinfectant-fixing solution for alginate impressions. It was found that the dimensional changes in impressions and stone cast roughness varied depending on not only the solution, but also the type of impression materials. As the dimensional changes and the stone cast roughness are the result of physicochemical interactions between the alginate gel and the solution, they may be influenced by both the gel and solution components.

In the previous paper ${ }^{7)}$, the volume change in a calcium alginate gel with no other constituents was measured in various types of solutions. Gels in monovalent metallic salt (potassium sulfate, sodium sulfate and sodium hypochlorite) solutions showed expansion, while they showed contraction in nonelectrolyte (glutaraldehyde) or divalent metallic salt (calcium chloride) solutions.

When gel expands or contracts in solutions, a transfer of substances between gel and solution may occur. Such a phenomenon makes it possible to clarify how the gel swelling/deswelling mechanism differs depending on the solution type. In this study, we focused on the ion concentration change of solutions containing gel, and examined 
the relationship between these concentrations and volume changes of the gels.

\section{MATERIALS AND METHODS}

\section{Materials}

The materials used in this experiment are shown in Table 1. Sodium alginate (300$400 \mathrm{cP}$ ) and calcium chloride were used to prepare the calcium alginate ( $\mathrm{Ca}-\mathrm{Alg})$ gel. As immersion solutions for gels, $\mathrm{CaCl}_{2}, \mathrm{~K}_{2} \mathrm{SO}_{4}$ and glutaraldehyde (GA) solutions, which were the fixing solution, plaster accelerator and disinfectant solutions, respectively, were used. In addition, $\mathrm{KCl}$ with the common cation to the plaster accelerator, a highly polar nonelectrolyte acetonitrile (AN), and a lower polar nonelectrolyte ethylene glycol (EG), were used for comparisons. The concentrations of the solutions were prepared to $0.03,0.3$ and $0.6 \mathrm{M}\left(0.015,0.15\right.$ and $0.3 \mathrm{M}$ for $\left.\mathrm{K}_{2} \mathrm{SO}_{4}\right)$ and other concentrations were used as needed.

\section{Preparation of calcium alginate gel}

A viscous sol of $1 \%$ sodium alginate was mounted in c.a. $2 \mathrm{~mm}$ thickness on an aluminum plate, $40 \mathrm{~mm}$ width and $60 \mathrm{~mm}$ length. It was soaked in $20 \% \mathrm{CaCl}_{2}$ solution for about $2 \mathrm{~min}$ for gelation. The resulting gel was separated from the plate and left in the $\mathrm{CaCl}_{2}$ solution for $6 \mathrm{hr}$ so that gelation would proceed sufficiently. After cutting them into pieces of $43 \times 30 \times 1 \mathrm{~mm}$, the gels were immersed in $1000 \mathrm{ml}$ of deionized water for 7 days to eliminate soluble salts included in the gels. The deionised water was exchanged three times during this period. The weight of the swollen and dried gels were approximately 0.8 and $0.02 \mathrm{~g}$, respectively.

\section{Measurement of ion concentration}

Desalted swollen gels, placed on a net-like bed of stainless steel wires so that the gel

Table 1 Materials used in this study

\begin{tabular}{|c|c|c|}
\hline Material & Code or formula & Lot No. \\
\hline \multicolumn{3}{|l|}{ Preparation of gel } \\
\hline Sodium alginate $(300-400 \mathrm{cP})^{1}$ & $\mathrm{Na}-\mathrm{Alg}$ & TPF7552 \\
\hline Calcium chloride ${ }^{2}$ & $\mathrm{CaCl}_{2}$ & $012 \mathrm{G} 1790$ \\
\hline \multicolumn{3}{|l|}{$\frac{\text { Preparation of aqueous solution }}{\text { (Nonelectrolytic) }}$} \\
\hline Ethylene Glycol ${ }^{2}$ & EG & $101 \mathrm{G} 1932$ \\
\hline Acetonitrile $^{2}$ & AN & $102 \mathrm{G} 1194$ \\
\hline $\begin{array}{l}\text { Glutaraldehyde }(25 \%)^{1} \\
\text { (Monovalent) }\end{array}$ & GA & ESM3059 \\
\hline Potassium chloride ${ }^{2}$ & $\mathrm{KCl}$ & 910 S2033 \\
\hline $\begin{array}{l}\text { Potassium sulfate }{ }^{2} \\
\text { (Divalent) }\end{array}$ & $\mathrm{K}_{2} \mathrm{SO}_{4}$ & 010G2045 \\
\hline Calcium chloride $^{2}$ & $\mathrm{CaCl}_{2}$ & $012 \mathrm{G} 1790$ \\
\hline
\end{tabular}

${ }^{1}$ Wako Pure Chemical Industries, Ltd., Osaka, Japan

${ }^{2}$ Kanto Chemical Co., Inc., Tokyo, Japan 
did not directly touch vessel bottom, were each immersed in $10 \mathrm{ml}$ solutions at room temperature for $20 \mathrm{~min}$. The ion concentration of each solution was determined using high performance liquid chromatography (LC-10ATVP, Shimadzu Corporation, Kyoto, Japan) with two columns, the Shim-pack IC-C1 and IC-A1 (Shimadzu Corporation, Kyoto, Japan) for cationic and anionic analyses, respectively. The mobile phases used for eluting the monovalent cation, divalent cation and anion were $5 \mathrm{mM}$ nitric acid, $4 \mathrm{mM}$ tartaric acid-2 $\mathrm{mM}$ ethylenediamine, and $2.5 \mathrm{mM}$ phthalic acid-2.4 $\mathrm{mM}$ tris(hydroxymethyl)aminomethan, respectively. The HPLC analyses were carried out with a flow rate of $1.5 \mathrm{ml} / \mathrm{min}$ at $40^{\circ} \mathrm{C}$ and the determination of each ion concentration was achieved by absolute calibration methods.

Measurement of volume changes in calcium alginate gels

According to the previous paper ${ }^{7)}$, the volume changes of desalted gels in each solution, $\Delta \mathrm{V} / \mathrm{V}_{0}$, were determined from the buoyancy change after 20 min's immersion. Here, the buoyancy change for gel due to elution of ions from the gel was negligible.

Statistical Analysis

Statistical analysis was made using the Student's t-test.

\section{RESULTS}

Changes in ion concentration

The changes of each ion concentration in the solutions after the Ca-Alg gel immersion are shown in Figs. 1-6. The positive values indicate an increase in ion concentration, while negative values indicate a decrease.

In EG solutions, little change in $\mathrm{Na}^{+}$and $\mathrm{Cl}^{-}$concentrations occurred (Fig. 1). The concentrations of $\mathrm{Ca}^{2+}$ eluted in $0.03,0.3$ and $0.6 \mathrm{M}$ EG solutions from the gel reached $4.4 \times 10^{-3}, 5.2 \times 10^{-3}$ and $5.0 \times 10^{-3} \mathrm{mM}$, respectively. However, the difference

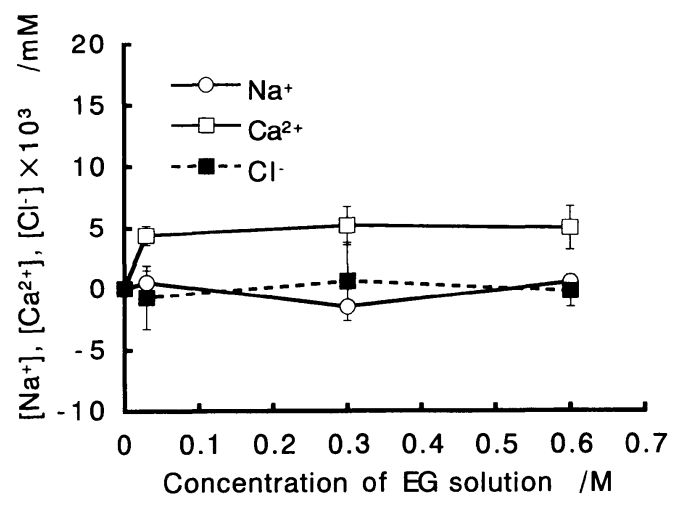

Fig. 1 Change in ionic concentration of the EG solution after $\mathrm{Ca}-\mathrm{Alg}$ gel immersion.

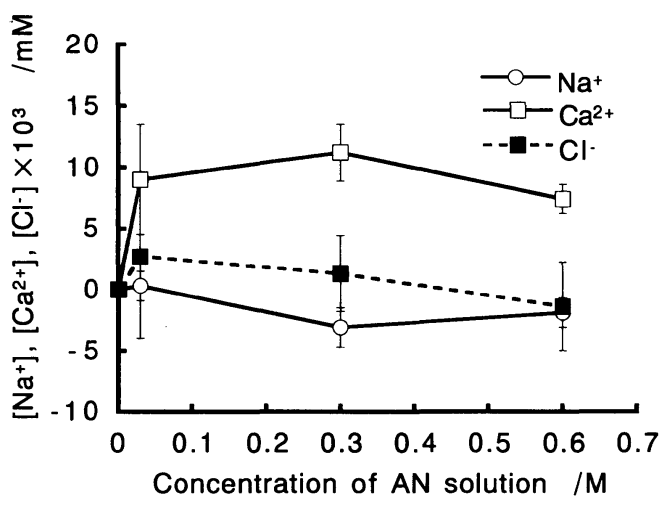

Fig. 2 Change in ionic concentration of the AN solution after $\mathrm{Ca}-\mathrm{Alg}$ gel immersion. 
in concentration between the solutions was not significant $(p>0.05)$.

In $\mathrm{AN}$ solutions, changes in $\mathrm{Na}^{+}$and $\mathrm{Cl}^{-}$concentration remained almost constant, as well as with the EG solution (Fig. 2). The increases in $\mathrm{Ca}^{2+}$ concentration in the $0.03,0.3$ and $0.6 \mathrm{M} \mathrm{AN}$ solutions were approximately twice those in the EG solutions, $9.0 \times 10^{-3}, 11.2 \times 10^{-3}$ and $7.4 \times 10^{-3} \mathrm{mM}$, respectively. There was no significant difference among the three AN solutions $(p>0.05)$.

In GA solutions, elution of $\mathrm{Cl}^{-}$was not observed at any solution concentration, while $\mathrm{Na}^{+}$concentration reduced with increases in GA concentration (Fig. 3). The elution of $\mathrm{Ca}^{2+}$ increased with GA concentration. The concentrations of $\mathrm{Ca}^{2+}$ eluted in the $0.03,0.3$ and $0.6 \mathrm{M}$ solutions were $26 \times 10^{-3}, 144 \times 10^{-3}$ and $300 \times 10^{-3} \mathrm{mM}$, respectively, indicating a linear increase with the concentration of the original solutions. The increase in $\mathrm{Ca}^{2+}$ in the $0.6 \mathrm{M}$ solution was approximately 40 times that in the AN solution.

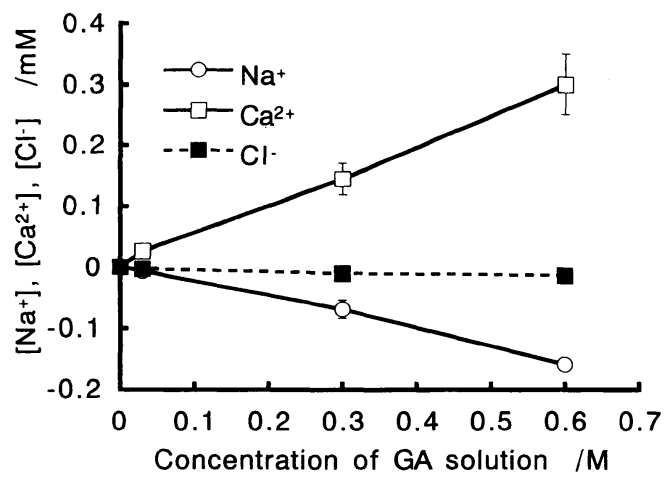

Fig. 3 Change in ionic concentration of the GA solution after $\mathrm{Ca}$-Alg gel immersion.

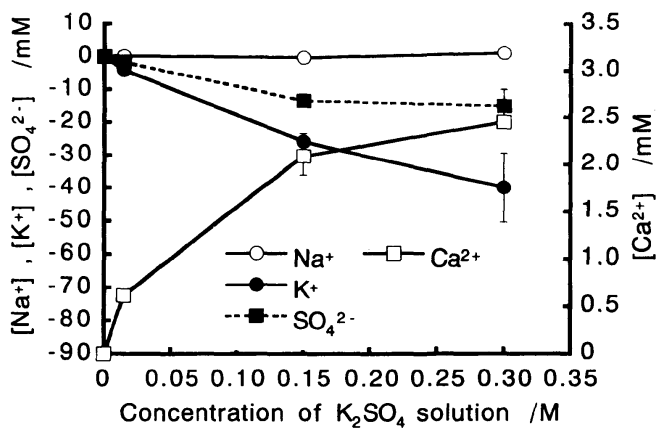

Fig. 5 Change in ionic concentration of the $\mathrm{K}_{2} \mathrm{SO}_{4}$ solution after $\mathrm{Ca}-\mathrm{Alg}$ gel immersion.

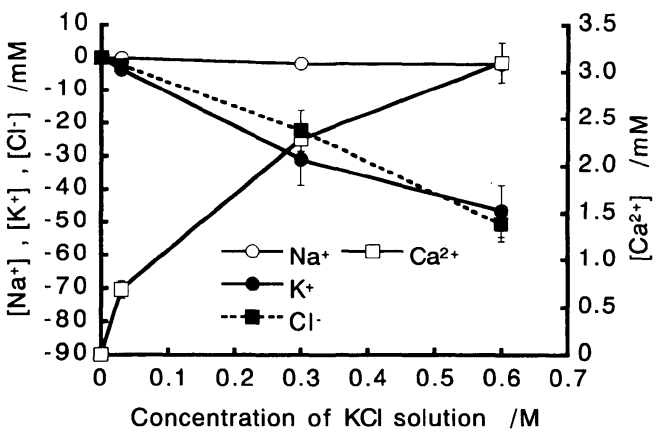

Fig. 4 Change in ionic concentration of the $\mathrm{KCl}$ solution after $\mathrm{Ca}-\mathrm{Alg}$ gel immersion.

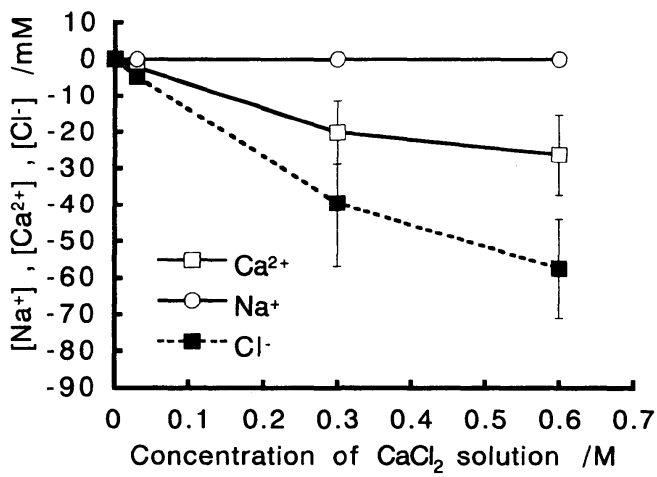

Fig. 6 Change in ionic concentration of the $\mathrm{CaCl}_{2}$ solution after $\mathrm{Ca}-\mathrm{Alg}$ gel immersion. 


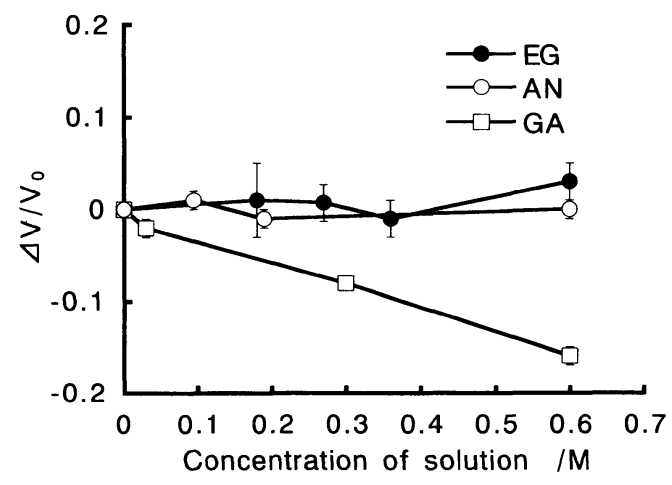

Fig. 7 Change in volume of the Ca-Alg gel in nonelectrolytic solutions.

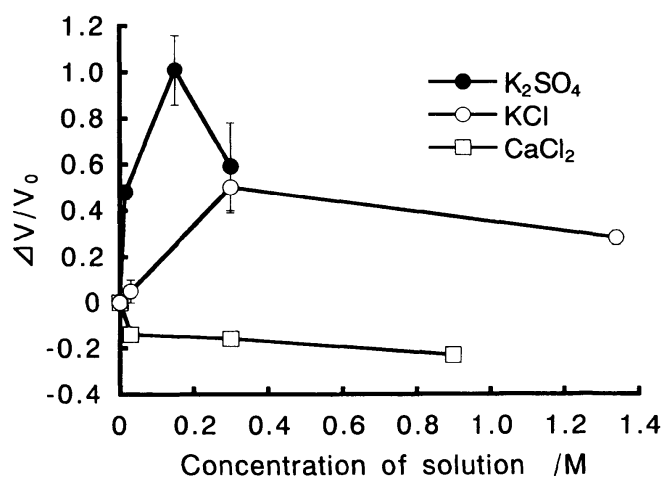

Fig. 8 Change in volume of the Ca-Alg gel in monovalent and divalent metallic salt solutions.

In the $\mathrm{KCl}$ solutions, $\mathrm{K}^{+}$and $\mathrm{Cl}^{-}$reduced by equivalent amount and the reduction was almost linearly proportional to the increase in $\mathrm{KCl}$ concentration (Fig. 4). On the other hand, the concentration of $\mathrm{Ca}^{2+}$ increased to $0.69,2.28$ and $3.10 \mathrm{mM}$, in the $0.03,0.3$ and $0.6 \mathrm{M}$ solutions, respectively. The increases were 10 to 26 times higher, compared to those of the GA solutions.

In the $\mathrm{K}_{2} \mathrm{SO}_{4}$ solutions, $\mathrm{K}^{+}$and $\mathrm{SO}_{4}{ }^{2-}$ reduced with increasing concentrations of $\mathrm{K}_{2} \mathrm{SO}_{4}$ (Fig. 5). The higher the solution concentration, the greater the reduction in each solution's ion concentration. The $\mathrm{SO}_{4}{ }^{2-}$ molar concentration was eventually approximately half that of $\mathrm{K}^{+}$. The concentrations of $\mathrm{Ca}^{2+}$ increased to $0.62,2.09$ and $2.45 \mathrm{mM}$, respectively, in the $0.015,0.15$ and $0.3 \mathrm{M}$ solutions, which was somewhat lower than those of the corresponding $\mathrm{KCl}$ solutions.

In the $\mathrm{CaCl}_{2}$ solutions, a change in concentration of $\mathrm{Na}^{+}$was not observed after the gel immersion (Fig.6). Both the concentration of $\mathrm{Ca}^{2+}$ and $\mathrm{Cl}^{-}$reduced with increases in the $\mathrm{CaCl}_{2}$ concentrations of the original solutions.

\section{Changes in calcium alginate gel volume}

The volume changes of gels, $\Delta \mathrm{V} / \mathrm{V}_{0}$, when immersed in the three types of nonelectrolytic solutions for $20 \mathrm{~min}$ are shown in Fig. 7. Less change was observed after immersion in the EG and AN solutions, while contraction occurred in the GA solution. The higher the concentration of GA, the greater the contraction.

The volume changes in monovalent and divalent metallic salt solutions are shown in Fig. 8. The gels swelled in $\mathrm{KCl}$ and $\mathrm{K}_{2} \mathrm{SO}_{4}$ solutions and contracted in the $\mathrm{CaCl}_{2}$ solution. The degree of swelling in the $\mathrm{KCl}$ and $\mathrm{K}_{2} \mathrm{SO}_{4}$ solutions was the greatest at $0.3 \mathrm{M}$ and $0.15 \mathrm{M}$, respectively, and they decreased at higher concentrations. The degree of gel shrinkage in the $\mathrm{CaCl}_{2}$ solution was $14 \%$ with the $0.03 \mathrm{M}$ solution, and this declined gradually with increases in the $\mathrm{CaCl}_{2}$ concentration of the original solutions. 


\section{DISCUSSION}

Dimensional changes in alginate impressions in solutions are caused by physical and chemical interactions between solutions and alginate gels. In the present study, the characteristic swelling/deswelling behaviors of alginate gels were observed when immersed in three types of solutions, namely, nonelectrolyte, monovalent or divalent salt solutions. In general, polymer gels consist of macromolecular network and the solvent molecules therein. Thus, the solvent in a gel can be extruded out, or uptaken from outside into the gel, according to the force balance between the network chain elasticity and osmotic pressure of the solvent. Moreover, this balance may be affected by the affinity between the polyelectrolyte and the solvent, and the osmotic pressure difference between the gel and the outer solution ${ }^{8)}$. The effects of these factors on the behavior of swelling/deswelling of each gel in each solution are discussed below.

\section{Gel volume changes in the nonelectrolytic solution}

In solutions up to $0.6 \mathrm{M}$ with nonelectrolytes $\mathrm{EG}$ and $\mathrm{AN}$, gel volume scarcely changed (Fig. 7). Also, there was little change in concentration of $\mathrm{Na}^{+}$and $\mathrm{Cl}^{-}$in these solutions, but there was a slight $\mathrm{Ca}^{2+}$ elution (Figs. 1 and 2). However, since the level of $\mathrm{Ca}^{2+}$ elution was very low, EG and AN were thought to be less influential on interactions between gel and solution. When the calcium alginate gel was immersed in solutions, an osmotic pressure difference between the desalted gel and immersion solution containing nonelectrolytes was generated.

Hence, we can assume that the solvent in the gel may move into the solution in order to cancel out the osmotic pressure difference, leading to gel contraction. In contrast to this speculation, no volume change occurred in the two nonelectrolytic solutions. Therefore, it is thought that the alginate gel may have no semipermeability for nonelectrolyte solutes and that the solvent can not move one way from gel to solution. A concentration difference between gel and solution would make solute species diffuse into the gel via a driving force until equilibrium was attained between them. In another nonelectrolyte GA solution, the gel volume decreased almost linearly with increases in GA concentration (Fig. 7). In addition, movement of traces of $\mathrm{Na}^{+}$into gel and elution of $\mathrm{Ca}^{2+}$ from the gel were observed (Fig. 3). The $\mathrm{Na}^{+}$in the GA solution was a constituent of the reagent $\left(0.01 \%\right.$ of $\mathrm{Na}^{+}$was detected in the $25 \%$ glutaraldehyde reagent). The eluted amount of $\mathrm{Ca}^{2+}$ into the $\mathrm{GA}$ solution was 3 to 40 times of that into the AN solution. The eluted $\mathrm{Ca}^{2+}$ probably arises from the calcium alginate gel. Thus, the GA solution is thought to function to liberate the calcium from the gel by scission of the crosslink. The gel, however, contracted in the GA solution, in contrast to the prediction that crosslink scission in the gel would lead to a reduction in the crosslinking density, thereby increasing the degree of swelling. This phenomenon could be attributed to another possible chemical interaction between the alginate gel and GA solution. Since the GA solution was acidic at $\mathrm{pH}$ 3 , hydronium ions would substitute both calcium ions in the crosslink, and sodium 
ions associating with the carboxylate residue, turning the carboxylate residue to an insoluble acid residue. On the other hand, based on an analogy of the reaction between hydroxyl groups in polymers and dialdehydes, reported by Yamauchi ${ }^{9)}$, it is predictable that hydroxyl groups in alginate may react with glutaraldehyde, a dialdehyde, to increase the crosslinking bond in the gel network, causing gel contraction. Additionally, it should be noted that the disinfectant potential of glutaraldehyde may be reduced by consuming part of the glutaraldehyde in the reaction with alginate.

\section{Gel volume changes in the monovalent metallic salt solution}

Gels immersed in solutions containing $\mathrm{K}^{+}, \mathrm{KCl}$ or $\mathrm{K}_{2} \mathrm{SO}_{4}$ swelled. The degree of swelling varied with the type and concentration of salts, with the maximum at $0.3 \mathrm{M}$ $\mathrm{K}^{+}$concentration, and decreasing at higher concentrations in the case of $\mathrm{KCl}$ solutions (Fig. 8). $\mathrm{K}^{+}, \mathrm{Cl}^{-}$and $\mathrm{SO}_{4}{ }^{2-}$ in the solution were trapped in the gel with simultaneous elution of $\mathrm{Ca}^{2+}$ from it (Figs. 4 and 5). As reported by Wang et al. ${ }^{10)}$, calcium in the alginate gel was replaced with $\mathrm{K}^{+}$and eluted into the solution. The experimental results indicated that $\mathrm{Ca}^{2+}$ elution increased with $\mathrm{KCl}$ concentration and the degree of swelling decreased with concentration levels above $0.3 \mathrm{M}$. More $\mathrm{Ca}^{2+}$ elution and less crosslinking density led to an increase in the swelling. We can not explain the reason for this contradiction. Presumably, cations other than $\mathrm{Ca}^{2+}$ entered the gel replacing the roles of $\mathrm{Ca}^{2+}$ cations as crosslink agents. However, it is thought that the greater difference in salt concentration between solution and gel results in solvent movement from the gel to the solution due to the osmotic pressure difference between them, and that the higher ionic strength in the gel acts on the morphological changes in inter-crosslinking molecular chains from extended form to shrunk form. The mechanism of swelling in the $\mathrm{K}_{2} \mathrm{SO}_{4}$ solution was considered to be almost the same as in $\mathrm{KCl}$, while the maximum degree of swelling in the $\mathrm{K}_{2} \mathrm{SO}_{4}$ solution was twice that in the $\mathrm{KCl}$ solution. Also, the crosslink scission in the $\mathrm{K}_{2} \mathrm{SO}_{4}$ solution progressed more than in the $\mathrm{KCl}$ solution. The gel immersed in the $\mathrm{K}_{2} \mathrm{SO}_{4}$ solution was evidently softer than that with $\mathrm{KCl}$, especially at high concentrations. Swelling of the gel in $\mathrm{K}_{2} \mathrm{SO}_{4}$ may have been due to the effect of anions, as well as cations. To discuss the effect of anions, $\mathrm{KCl}$ and $\mathrm{K}_{2} \mathrm{SO}_{4}$ solutions with the same concentration of $\mathrm{K}^{+}$are compared with each other below, assuming that their degrees of ionization were equal. The concentration of $\mathrm{Ca}^{2+}$ in the $0.3 \mathrm{M} \mathrm{K}_{2} \mathrm{SO}_{4}$ solution after immersing in the gel (Fig. 5) was obviously lower than that in the $0.6 \mathrm{M} \mathrm{KCl}$ solution (Fig. 4). Based on the difference in solubility between $\mathrm{CaSO}_{4}$ and $\mathrm{CaCl}_{2}$, this suggested that $\mathrm{SO}_{4}{ }^{2-}$ facilitated dissociation of $\mathrm{Ca}^{2+}$ in the gel more extensively than $\mathrm{Cl}^{-}$.

\section{Gel volume changes in the divalent metallic salt solution}

When immersed in the $\mathrm{CaCl}_{2}$ solution, gel trapped $\mathrm{Ca}^{2+}$ from the solution and contracted. After 20 min's immersion, the concentration in the $\mathrm{Ca}^{2+}$ in $0.03,0.3$ and 0.6 $\mathrm{M} \mathrm{CaCl}_{2}$ solutions decreased by $2.2,20.1$ and $26.2 \mathrm{mM}$, respectively (Fig. 6 ), followed 
by gel contraction of 14,16 , and $23 \%$, respectively ( $23 \%$ contraction was observed in the $0.9 \mathrm{M}$ solution) (Fig. 8). As is well known, $\mathrm{Ca}^{2+}$ reacts with residual carboxylate groups in alginate gels to increase crosslinking density, thus facilitating gel contraction. Here, to estimate how many $\mathrm{Ca}^{2+}$ ions trapped in the gel were relevant to the crosslinking of residual carboxylate groups, the amount of $\mathrm{Ca}^{2+}$ required for crosslinking was calculated. The weight of a piece of an alginate gel specimen made from $1 \%$ sodium alginate sol was c.a. $0.8 \mathrm{~g}$. Assuming that $1 \%$ of the gel specimen corresponded roughly to the weight of sodium alginate, the molarity of mannuronic acid residues was $4.04 \times 10^{-2} \mathrm{mM}$. The crosslinking of alginate through mannuronic acid residues by $\mathrm{Ca}^{2+}$ can be expressed as:

$$
2(\mathrm{Na}-\mathrm{Alg})_{\text {residue }}+\mathrm{Ca}^{2+} \rightarrow\left(\mathrm{Ca}-\mathrm{Alg}_{2}\right)_{\text {crosslink }}+2 \mathrm{Na}^{+}
$$

Accordingly, $\mathrm{Ca}^{2+}$ ions of a half mole of mannuronic acid residues, $2.02 \times 10^{-2} \mathrm{mM}$, were required for crosslinking all residues. On the other hand, the amount of $\mathrm{Ca}^{2+}$ ions trapped in the gel from $10 \mathrm{ml}$ each of the $0.03,0.3$ and $0.6 \mathrm{M}$ solutions were $2.2 \times 10^{-2}, 20.1 \times 10^{-2}$ and $26.2 \times 10^{-2} \mathrm{mM}$, respectively. These estimates showed that an excess amount of $\mathrm{Ca}^{2+}$ ions were trapped in the gel. In general, multifunctional alginates have lower gel points, that is, only a low percentage of $\mathrm{Ca}^{2+}$ ions is stoichiometrically needed for gel formation. Thus, it is thought that most carboxylate residues in the gel remain unreacted, and that part of the uncrosslinked residues, which are available for additional crosslinking of the swollen gel due to the desalting process, can react with $\mathrm{Ca}^{2+}$ ions leading to additional contraction. This is consistent with the evidence that the dependence of additional gel contraction on the concentration of the immersing solution was not very high, as shown in Fig. 8.

From this study, the mechanism of swelling/deswelling of calcium alginate gels in nonelectrolyte and electrolyte solutions, which were clinically used as fixing and disinfectant solutions, was clarified. This study contributes to the development of dimensionally stable fixing and disinfectant treatment solutions for alginate impressions.

\section{REFERENCES}

1) Ichimaru, T., Saito, S. and Kon, R.: Disinfecting-fixing solutions for alginate impression, J J Dent Mater 13(4) : 331-339, 1994. (in Japanese)

2) Ichimaru, T., Saito, S. and Nagata, K.: Disinfectant-fixing combined solution for alginate impression using sodium hypochlorite, Dent $J$ Iwate Med Univ $21: 29-40,1996$. (in Japanese)

3) Ichimaru, T., Saito, S. and Kon, R.: Dimensional stability of alginate impressions in a disinfectant-fixing solution and changes in $\mathrm{pH}$ and ion concentration of the solution, $J J$ Dent Mater 18(5): 339-346, 1999. (in Japanese)

4) Saito, S. and Ichimaru, T.: Effects of surfactant treatment for alginate impression material on the surface roughness of dental stone, $J J$ Dent Mater 11(4):570-577, 1992. (in Japanese)

5) Ichimaru, T. and Saito, S.: Mixing and/or fixing treatment with disinfectant solutions for alginate impression materials, $D E 106: 16-19$, 1993. (in Japanese)

6) Saito, S.: Surface roughness of stone cast set against alginate impression, $J J$ Dent 
Mater 14(6) : 613-628, 1995. (in Japanese)

7) Saito, S., Ichimaru, T. and Araki, Y.: Factors affecting dimensional instability of alginate impressions during immersion in the fixing and disinfectant solutions, Dent Mater $J$ 17(4) : 294-300, 1998.

8) Masuda, H.: Superabsorbent polymers, 1st ed., Kyoritsu Publishing Co., Tokyo, 1988, pp.12-23. (in Japanese)

9) Yamauchi, A.: Preparation and structure of gel, Tanpakushitu Kakusan Kouso 22(13): 1418-1423, 1977. (in Japanese)

10) Wang, X. and Spencer, H. G.: Calcium alginate gels: formation and stability in the presence of an inert electrolyte, Polymer 39(13): 2759-2764, 1998. 